\title{
Self-similar spherically symmetric wave maps coupled to gravity
}

\author{
Piotr Bizoń* and Arthur Wasserman ${ }^{\dagger}$ \\ *Institute of Physics, Jagellonian University, Kraków, Poland \\ ${ }^{\dagger}$ Department of Mathematics, University of Michigan, Ann Arbor, Michigan
}

November 6, 2018

\begin{abstract}
We investigate spherically symmetric continuously self-similar (CSS) solutions in the $S U(2)$ sigma model coupled to gravity. Using mixed numerical and analytical methods, we provide evidence for the existence (for small coupling) of a countable family of regular CSS solutions. This fact is argued to have important implications for the ongoing studies of black hole formation in the model.
\end{abstract}

\section{Introduction}

Self-similar solutions of Einstein's equations have been extensively studied in general relativity (see [1] for the recent review and vast bibliography of this subject). They are interesting for several reasons. First, under the assumption of self-similarity Einstein's equations simplify considerably and sometimes (in spherical symmetry, for instance) reduce to a system of ordinary differential equations. This enables one to study them using dynamical systems methods and in some cases it is even possible to find explicit solutions. Second, self-similar solutions exhibit a well-defined singular behaviour at the blow-up. This makes them relevant in the studies of singularity formation; in fact, most of the examples of naked singularities in the literature involve self-similar solutions. Third, in some situations solutions of the Cauchy problem starting from generic initial data evolve asymptotically to self-similar form, in other words, self-similar solutions can play the role of attractors. Finally, the recent surge of interest in self-similar solutions is due to the fact that they appear as critical solutions at the threshold for black hole formation in gravitational collapse.

Such reasons motivated also this paper which, in a sense, is an extension of [2] where selfsimilar wave maps from Minkowski spacetime into the 3-sphere were studied. It was proved in [2] that there exists a countable family of regular (by regularity we mean analyticity below the Cauchy horizon of the singularity) CSS solutions, labeled by a nonnegative integer $n$ (a nodal number). It was also shown that the $n$th solution has exactly $n$ unstable modes. The role of these solutions in the dynamical evolution was investigated in [3] where it was shown that: (i) the $n=0$ solution determines a universal asymptotics of singularity formation, (ii) the $n=1$ solution appears as a critical solution at the threshold for singularity formation (see also (4). Here, we generalize [2] by turning on gravity, that is, we consider wave maps with the 3 -sphere as the target and a domain manifold which is not Minkowski but a spacetime satisfying the Einstein equations with the $S U(2)$ sigma field as the source. The coupling of gravity is parametrized by a dimensionless coupling constant $\alpha$ (so that $\alpha=0$ corresponds to gravity being turned off). Since the coupling does not break scale invariance, it is natural to ask if the $\alpha=0$ CSS solutions constructed in 2], persist for nonzero $\alpha$. A positive answer to this question is the main result of 
our paper. More precisely, we show that the $n$th CSS solution which is analytic within the past light cone of the singularity (in what follows we will refer to the past and future light cones of the singularity as to the past and future self-similarity horizons (SSH)), persists up to $\alpha=1 / 2$. However, this solution is regular (that is, analytic up to the future SSH) only for $\alpha<\alpha_{n}$, where $\left\{\alpha_{n}\right\}$ is an increasing sequence bounded above by $1 / 2$. For $\alpha_{n}<\alpha<1 / 2$, the past SSH of the $n$th solution is surrounded by a spacelike apparent horizon beyond which the solution cannot be smoothly continued. Although we have not studied linear stability, it seems plausible that the stability properties of solutions for small $\alpha$ are the same as for $\alpha=0$. Assuming this, the $n=0$ solution (for $\alpha<\alpha_{0} \approx 0.0688$ ) is a generic naked singularity, while the $n=1$ solution (for $\alpha<\alpha_{1} \approx 0.1518$ ) is a codimension-one naked singularity and the candidate for a critical solution.

The CSS solutions constructed here should be relevant for the character of type II critical collapse in the model which is currently being investigated by Husa, Lechner, Pürrer, Thornburg, and Aichelburg (HLPTA). Their first report of progress [5] shows discretely self-similar (DSS) behaviour at the threshold for black hole formation for $\alpha \geq 0.18$ and indicates that DSS disappears for small $\alpha$. It is natural to expect that for $\alpha<\alpha_{1}$ the $n=1$ CSS solution takes over as a critical solution. Preliminary results of HLPTA seem to confirm this expectation [6]. A mechanism of the DSS/CSS changeover is not yet understood, however. We anticipate that the standard type II CSS critical behaviour will be observed only in the range $\alpha_{0}<\alpha<\alpha_{1}$. For $\alpha<\alpha_{0}$ we expect qualitatively the same behaviour as in the flat spacetime, where the $n=1$ solution sits at the threshold for the $n=0$ naked singularity formation.

The rest of the paper is organized as follows. In section 2 we define the model and derive the field equations. In section 3 we construct a countable family of CSS solutions which are analytic between the center and the past SSH. An extension of these solutions beyond the past SSH is determined in section 4 . In section 5 we speculate about the role of regular CSS solutions in the dynamical evolution.

\section{Field Equations}

Let $X: M \rightarrow N$ be a map from a spacetime $\left(M, g_{a b}\right)$ into a Riemannian manifold $\left(N, G_{A B}\right)$. Wave maps coupled to gravity are defined as extrema of the action

$$
S=\int_{M}\left(\frac{R}{16 \pi G}+L_{W M}\right) d v_{g}
$$

with the Lagrangian density

$$
L_{W M}=-\frac{f_{\pi}^{2}}{2} g^{a b} \partial_{a} X^{A} \partial_{b} X^{B} G_{A B}
$$

Here $G$ is Newton's constant and $f_{\pi}^{2}$ is the wave map coupling constant. Remarkably, in the "physical" case of $3+1$ dimensional spacetime (to which our paper is confined), the coupling of gravity does not break scale invariance but introduces the dimensionless coupling constant $\alpha=4 \pi G f_{\pi}^{2}$. The field equations derived from (1) are the wave map equation

$$
\square_{g} X^{A}+\Gamma_{B C}^{A}(X) \partial_{a} X^{B} \partial_{b} X^{C} g^{a b}=0,
$$

where $\Gamma_{B C}^{A}(X)$ are the Christoffel symbols of the target metric $G_{A B}$ and $\square_{g}$ is the d'Alembertian associated with the metric $g_{a b}$, and the Einstein equations $R_{a b}-\frac{1}{2} g_{a b} R=8 \pi G T_{a b}$ with the stressenergy tensor

$$
T_{a b}=f_{\pi}^{2}\left(\partial_{a} X^{A} \partial_{b} X^{B}-\frac{1}{2} g_{a b}\left(g^{c d} \partial_{c} X^{A} \partial_{d} X^{B}\right)\right) G_{A B} .
$$


The target manifold is taken as the three-sphere $S^{3}$ with the standard metric in polar coordinates $X^{A}=(F, \Theta, \Phi)$

$$
G_{A B} d X^{A} d X^{B}=d F^{2}+\sin ^{2} F\left(d \Theta^{2}+\sin ^{2} \Theta d \Phi^{2}\right) .
$$

For the domain manifold we assume spherical symmetry and use Schwarzschild coordinates

$$
g_{a b} d x^{a} d x^{b}=-e^{-2 \delta} A d t^{2}+A^{-1} d r^{2}+r^{2}\left(d \theta^{2}+\sin ^{2} \theta d \phi^{2}\right),
$$

where $\delta$ and $A$ are functions of $(t, r)$. Next, we assume that the wave maps are corotational, that is

$$
F=F(t, r), \quad \Theta=\theta, \quad \Phi=\phi .
$$

Eq.(3) reduces then to the single semilinear wave equation

$$
\square_{g} F-\frac{\sin (2 F)}{r^{2}}=0
$$

where

$$
\square_{g}=-e^{\delta} \partial_{t}\left(e^{\delta} A^{-1} \partial_{t}\right)+\frac{e^{\delta}}{r^{2}} \partial_{r}\left(r^{2} e^{-\delta} A \partial_{r}\right)
$$

and the Einstein equations are

$$
\begin{aligned}
\partial_{t} A & =-2 \alpha r A\left(\partial_{t} F\right)\left(\partial_{r} F\right) \\
\partial_{r} \delta & =-\alpha r\left(\left(\partial_{r} F\right)^{2}+A^{-2} e^{2 \delta}\left(\partial_{t} F\right)^{2}\right) \\
\partial_{r} A & =\frac{1-A}{r}-\alpha r\left(A\left(\partial_{r} F\right)^{2}+A^{-1} e^{2 \delta}\left(\partial_{t} F\right)^{2}+2 \frac{\sin ^{2} F}{r^{2}}\right) .
\end{aligned}
$$

These equations are invariant under dilations $(t, r) \rightarrow(\lambda t, \lambda r)$ so it is natural to look for continuously self-similar (CSS) solutions, that is solutions which are left invariant by the action of the homothetic Killing vector $K=t \partial_{t}+r \partial_{r}$. To study such solutions it is convenient to use similarity variables $\rho=r /(-t)$ and $\tau=-\ln (-t)$. Then $K=-\partial_{\tau}$, so CSS solutions do not depend on $\tau$. Assuming this and using an auxiliary function $z=e^{\delta} \rho / A$, we reduce Eqs.(8-12) to the system of ordinary differential equations (where prime is $d / d \rho$ )

$$
\begin{aligned}
F^{\prime \prime} & =\frac{2}{\rho} F^{\prime}-\alpha\left(1+z^{2}\right) \rho{F^{\prime}}^{3}-\frac{\sin (2 F)}{A \rho^{2}\left(1-z^{2}\right)}=0, \\
A^{\prime} & =-2 \alpha \rho A{F^{\prime}}^{2}, \\
\rho z^{\prime} & =z\left(1+\alpha\left(1-z^{2}\right) \rho^{2}{F^{\prime}}^{2}\right), \\
\rho A^{\prime} & =1-A-\alpha\left(\rho^{2} A\left(1+z^{2}\right){F^{\prime}}^{2}+2 \sin ^{2} F\right) .
\end{aligned}
$$

The combination of (14) and (16) yields the constraint

$$
1-A-2 \alpha \sin ^{2} F+\alpha A \rho^{2}{F^{\prime}}^{2}\left(1-z^{2}\right)=0 .
$$

In what follows, we will be solving Eqs.(13-15). The constraint (17), which is the first integral of these equations, could be used to eliminate $A$ from (13) but we find more convenient not to do so. The system (13-15) has a fixed singularity at the center $\rho=0$ and moving singularities 
at points where $z(\rho)= \pm 1$ and/or $A(\rho)=0$. In terms of the similarity coordinate $\rho$, the metric (6) takes the form

$$
d s^{2}=A^{-1}\left(1-z^{-2}\right) \rho^{2} d t^{2}+2 A^{-1} t \rho d t d \rho+A^{-1} t^{2} d \rho^{2}+t^{2} \rho^{2}\left(d \theta^{2}+\sin ^{2} \theta d \phi^{2}\right),
$$

hence the hypersurfaces $z= \pm 1$ are null (provided that $A>0$ ). After [7] we will refer to these characteristic hypersurfaces as to the self-similarity horizons (SSH). The first $\rho_{0}$ where $z\left(\rho_{0}\right)=1$ is the locus of the past light cone of the singularity at the origin $(t=0, r=0)$ and we will call it the past SSH. We use the remaining coordinate freedom to locate it at $\rho_{0}=1$, that is $z(1)=1$. In the next section we will analyze the system (13-15) below the past SSH, that is for $0 \leq \rho \leq 1$.

\section{From the center to the past self-similarity horizon}

Expanding about $\rho=1$, we get a one-parameter family of solutions of Eqs.(13-15)

$$
F(\rho) \sim \frac{\pi}{2}+b(\rho-1), \quad z(\rho) \sim 1+(\rho-1), \quad A(\rho) \sim 1-2 \alpha-2 \alpha(1-2 \alpha) b^{2}(\rho-1) .
$$

We shoot these initial data towards $\rho=0$ and adjust the parameter $b$ so that the solution satisfies the regularity condition at the center,

$$
F(0)=0, \quad z(0)=0, \quad A(0)=1 .
$$

We find numerically that for each $\alpha<1 / 2$, there is an infinite sequence $\left\{b_{n}\right\}, n=0,1, \ldots$, such that the corresponding solutions $\left(F_{n}, A_{n}, z_{n}\right)$ satisfy the boundary conditions (19) and (20). The index $n$ denotes the number of solutions of the equation $F_{n}(\rho)=\pi / 2$ on the interval $0<\rho<1$. In the limit $\alpha \rightarrow 0$, the $n$th solution tends uniformly to the corresponding $\alpha=0$ solution. In the limit $\alpha \rightarrow 1 / 2$, the parameters $b_{n}(\alpha)$ diverge to infinity indicating that regular solutions disappear for $\alpha \geq 1 / 2$. Fig. 1 shows how the shooting parameters $b_{n}$ depend on $\alpha$ for $n=0$ and $n=1$. In Fig. 2 we plot the $n=0$ and $n=1$ solutions for $\alpha=0.1$.

To summarize, we claim that for each $\alpha<1 / 2$ there exists a countable family of CSS solutions which are analytic at the center and at the past SSH. The structure of these solutions is basically the same as for $\alpha=0$. Qualitatively new things happen beyond the past SSH and now we turn our attention to this much more interesting region.

\section{Beyond the past self-similarity horizon}

For $\alpha=0$ it was easy to show that all solutions starting at $\rho=1$ with initial values (19) remain regular for all $\rho>1$. As we shall see below this is still true for $\alpha>0$ provided that $b$ is sufficiently small (note, however, that the metric function $A(\rho)$ is monotone decreasing, so $A(\infty)<1$, which means that the geometry is not asymptotically flat but conical). However, if $b$ is large, the function $z(\rho)$ is not monotone increasing for all $\rho>1$ and a "sonic point" develops for some finite $\rho_{B}>1$, that is $\lim _{\rho \rightarrow \rho_{B}} z(\rho)=1$. We stress that this "sonic point" is not a SSH but an apparent horizon because $\lim _{\rho \rightarrow \rho_{B}} A(\rho)=0$ as follows easily from (14) and (15). These two kinds of behaviour are illustrated in Fig. 2.

Note that $\rho=\infty$ corresponds to the hypersurface $(t=0, r>0)$ so in order to analyze the global behaviour of solutions (for $t>0$ ) we need to go "beyond $\rho=\infty$ ". To this end we define a new coordinate $x$ by

$$
\frac{d}{d x}=\rho z \frac{d}{d \rho}, \quad x(\rho=1)=0
$$


We also define an auxiliary function $w(x)=1 / z(\rho)$. In these new variables, the past SSH where $w=1$ is at $x=0$, while the future $\mathrm{SSH}$ (which nota bene is a Cauchy horizon) is located at some $x_{A}>0$ where $w\left(x_{A}\right)=-1$.

In terms of $x$ and $w$, Eqs.(13-15) become autonomous (where now prime is $d / d x$ )

$$
\begin{aligned}
w^{\prime} & =-1+\alpha\left(1-w^{2}\right){F^{\prime}}^{2}, \\
A^{\prime} & =-2 \alpha A w{F^{\prime}}^{2}, \\
\left(A F^{\prime}\right)^{\prime} & =\frac{\sin (2 F)}{w^{2}-1} .
\end{aligned}
$$

The constraint (17) becomes

$$
1-A-2 \alpha \sin ^{2} F+\alpha A{F^{\prime}}^{2}\left(w^{2}-1\right)=0 .
$$

From (19) the initial conditions at $x=0$ are

$$
F(x) \sim \frac{\pi}{2}+b x, \quad w(x) \sim 1-x, \quad A(x) \sim 1-2 \alpha-2 \alpha(1-2 \alpha) b^{2} x .
$$

We already know from the previous section that for each $\alpha<1 / 2$ there is an infinite sequence $\left\{b_{n}\right\}$ determining solutions which are regular for all $x \leq 0$ (note that $\rho=0$ corresponds to $x=-\infty$ ). Although these solutions are completely fixed at the past SSH by the requirement of analyticity at the center, in order to understand their behaviour for $x>0$ it is helpful to drop this requirement temporarily and consider solutions that start from generic initial conditions (26). Of course, such solutions in general are not analytic at the center.

We first shall show that if $b$ is sufficiently small, then the solution remains regular up to the future SSH. To see this, let $f=(F-\pi / 2) / b$. Then, in the limit $b \rightarrow 0$, Eqs.(22-24) reduce to

$$
\begin{aligned}
w^{\prime} & =-1+\alpha\left(1-w^{2}\right) b^{2}{f^{\prime}}^{2} \rightarrow-1, \\
A^{\prime} & =-2 \alpha A w b^{2}{f^{\prime}}^{2} \rightarrow 0, \\
\left(A f^{\prime}\right)^{\prime} & =-\frac{\sin (2 b f)}{b\left(w^{2}-1\right)} \rightarrow-\frac{2 f}{w^{2}-1},
\end{aligned}
$$

with the initial conditions

$$
f(0)=0, \quad f^{\prime}(0)=1, \quad w(0)=1, \quad A(0)=1-2 \alpha .
$$

The limiting equations (27) and (28) are solved by $w=1-x$ and $A=1-2 \alpha$. Substituting these solutions into (29) we get the equation

$$
(1-2 \alpha) f^{\prime \prime}+\frac{2 f}{x(x-2)}=0,
$$

whose solution is given by the hypergeometric function $f(x)=x_{2} F_{1}[(1-i \sqrt{7}) / 2,(1+i \sqrt{7}) / 2,2, x / 2]$. Since solutions of Eqs.(22-24) are continuous in $b$ and $x$, by uniform continuity on compact intervals, the solutions with sufficiently small $b$ will tend to $w=-1$.

Asymptotic analysis at the future $\mathrm{SSH}, x_{A}$, yields the following leading order behaviour (where $\left.y=x_{A}-x\right)$

$$
w \sim-1+y, \quad A \sim A_{0}-2 \alpha A_{0} c^{2} y \ln ^{2}(y), \quad F \sim F_{0}-c y \ln (y),
$$

where $A_{0}=1-2 \alpha \sin ^{2} F_{0}, c=-\sin \left(2 F_{0}\right) / 2 A_{0}$, and $F_{0}$ is a free parameter. Thus, a solution that is analytic at the past SSH, generically will be only $C^{0}$ at the future SSHI. Nevertheless,

\footnotetext{
${ }^{1}$ If $F_{0}$ is an integer multiple of $\pi / 2$, then the singular log terms in (32) are absent and the solution is smooth at the future SSH. One can show that this happens for discrete values of $b$, which means that there exist solutions which are analytic both at the past and the future SSH. Of course, for generic $\alpha$ these solutions are singular at the center.
} 
we checked that with the asymptotic behaviour (32) all curvature invariants remain finite as $x \rightarrow x_{A}$. We interpret this somewhat surprising fact as an indication that the singularity at the origin is naked?.

Next, we shall show that solutions with large $b$ develop a "sonic point" for some finite $x_{B}>0$, that is, $\lim _{x \rightarrow x_{B}} w(x)=1$ and $\lim _{x \rightarrow x_{B}} A(x)=0$. This time, we define the variables

$$
\xi=b^{2} x, \quad h(\xi)=b^{2}(1-w(x)), \quad s(\xi)=b\left(F(x)-\frac{\pi}{2}\right) .
$$

Then, in the limit $b \rightarrow \infty$, Eqs.(22-24) reduce to (where now prime is $d / d \xi$ )

$$
\begin{aligned}
h^{\prime} & =1-\alpha h\left(2-\frac{h}{b^{2}}\right) s^{\prime 2} \rightarrow 1-2 \alpha h s^{\prime 2}, \\
A^{\prime} & =-2 \alpha A\left(1-\frac{h}{b^{2}}\right) s^{\prime 2} \rightarrow-2 \alpha A s^{\prime 2}, \\
\left(A s^{\prime}\right)^{\prime} & =\frac{\sin (2 s / b)}{b h\left(2-h / b^{2}\right)} \rightarrow 0,
\end{aligned}
$$

with the initial conditions

$$
h(0)=0, \quad A(0)=1-2 \alpha, \quad s(0)=0, \quad s^{\prime}(0)=1 .
$$

It follows from (36) and (37) that, in the limit $b \rightarrow \infty, A s^{\prime}=1-2 \alpha$. Plugging this into (34) and (35), and using (37), we get the limiting solution

$$
A(\xi)=(1-2 \alpha) \sqrt{1-4 \alpha \xi} \text { and } h(\xi)=\frac{1}{2 \alpha} \sqrt{1-4 \alpha \xi}(1-\sqrt{1-4 \alpha \xi}) .
$$

This solution develops a "sonic point" at $\xi=1 / 4 \alpha$. Again, by uniform continuity on compact intervals, we conclude that for solutions of Eqs.(22-24) with large $b$ (and nonzero $\alpha$ ), the function $w(x)$ attains a minimum and then tends to 1 at some $x \rightarrow x_{B}$, while the function $A(x)$ drops to zero at $x_{B}$. The leading order behaviour at the apparent horizon at $x_{B}$ is

$$
w \sim 1-a \sqrt{x_{B}-x}, \quad A \sim d \sqrt{x_{B}-x}, \quad F \sim F_{B}+\frac{1}{\sqrt{\alpha}} \sqrt{x_{B}-x},
$$

where the positive parameters $F_{B}, a, d$ are constrained by the relationship $1-2 \alpha \sin ^{2} F_{B}-a d / 2=$ 0 . Substituting this asymptotic behaviour into the metric (18), one can readily verify that the hypersurface $x=x_{B}$ is spacelike.

Thus, solutions which start from the past SSH with initial conditions (26), tend in finite "time" to $w=-1$ if $b$ is small, or to $w=+1$ if $b$ is large. In what follows, we will refer to these two behaviours as to type A and type B solutions, respectively. The solutions of type A obviously form an open set and we believe (but have no proof yet) that the same is true for the solutions of type B. If so, there must exist solutions which are not of type A or B, call them type $\mathrm{C}$ solutions. By definition, type $\mathrm{C}$ solutions remain in the strip $w \in(-1,1)$ which implies that they exist for all $x$ (because one can easily show that solutions can go singular only if $w \rightarrow \pm 1$ ).

Our numerical analysis of the structure of types A, B, and C can be summarized as follow 3 . For a given $\alpha$, there exists a critical value $b^{*}(\alpha)$ such that solutions with $b<b^{*}$ are of type A,

\footnotetext{
${ }^{2} \mathrm{~A}$ similar behaviour for CSS solutions of the Einstein-axion-dilaton equations was observed by Eardley, Hirschmann, and Horne 8].

${ }^{3}$ To simplify the exposition, we are cheating at this point. We have evidence that for large values of $\alpha(\sim 0.42)$ the transition between types $\mathrm{A}$ and $\mathrm{B}$ occurs not at a single point but in a narrow interval $\left(b_{\min }^{*}, b_{\max }^{*}\right)$. We suspect that this interval, which can be thought of as the boundary between the basins of attractions of type A and $\mathrm{B}$ behaviours, has a fractal structure. This intriguing fact, suggesting perhaps a chaotic behaviour, is under investigation and will be described elsewhere.
} 
solutions with $b>b^{*}$ are of type $\mathrm{B}$, and the solution with $b=b^{*}$ is of type $\mathrm{C}$. Thus, we have a bistable behaviour with two generic final states A and B, and the separatrix C. Although the precisely critical initial condition $b=b^{*}$ cannot be prepared numerically, in order to figure out a behaviour of the type $\mathrm{C}$ solution it is sufficient (due to continuous dependence of solutions on initial conditions) to determine the flow of nearly critical initial conditions $b=b^{*} \pm \epsilon$. As is shown in Fig. 3, before the marginally critical type A and B solutions tend to $w= \pm 1$, they exhibit a long transient almost periodic behaviour. This leads us to conjecture that the type $\mathrm{C}$ solution is asymptotically periodic. The periodic intermediate attractor is clearly seen on the $(w, A)$ plane.

The complete discussion of periodic solutions would take us too far afield, so we postpone it to the subsequent paper. Here, let us only point out that the behaviour of CSS solutions outside the past SSH resembles some aspects of the DSS type II critical behaviour at the threshold for black hole formation. Actually, it can be interpreted as a poor ODE version of this phenomenon. The type A and B solutions are the analogues of dispersive and black-hole solutions, respectively. The type $\mathrm{C}$ solution is the analogue of the critical evolution which tends asymptotically to the critical periodic solution (which is the analogue of the choptuon). We even have the analogue of the black-hole-mass scaling. Namely, let $\lambda$ be the unstable eigenvalue (that is $\operatorname{Re}(\lambda)>0$ ) around the periodic solution. Then the distance of a nearly critical flow (corresponding to $b=b^{*}+\epsilon$ ) from the separatrix $\mathrm{C}$ is proportional to $\epsilon \exp (\operatorname{Re}(\lambda) x)$, which means that the length of the transient periodic phase is proportional to $-\frac{1}{\operatorname{Re}(\lambda)} \ln |\epsilon|$. The locus of the future SSH $x_{A}$ (for the marginally critical type A solutions) and the locus of an apparent horizon $x_{B}$ (for the marginally critical type B solutions) scale in the same manner (see Fig. 4). This leading order scaling law is decorated in the next order by periodic wiggles with period $\operatorname{Re}(\lambda) T$. The origin of these wiggles is basically the same as in the case of black-hole mass scaling in the type II DSS critical collapse [7]. In passing, we remark that the periodic solutions are nonperturbative in $\alpha$-in the limit $\alpha \rightarrow 0$, the period and the amplitude of oscillations go to zero.

Now, we return to the countable family of CSS solutions constructed in section 3 and finally determine their extension beyond the past SSH. As follows from the above discussion, for a given $\alpha$, the behaviour of the $n$th solution outside the past SSH depends on whether $b_{n}$ is smaller or larger than $b^{*}$ : the solutions with $b_{n}<b^{*}$ are of type A (and therefore regular in our terminology), while the solutions with $b_{n}>b^{*}$ are of type $\mathrm{B}$. The function $b^{*}(\alpha)$ is monotone decreasing and $b^{*}(\alpha) \rightarrow \infty$ as $\alpha \rightarrow 0$, while the functions $b_{n}(\alpha)$ are monotone increasing, hence we obtain an infinite sequence $\left\{\alpha_{n}\right\}$ defined by the intersections $b^{*}\left(\alpha_{n}\right)=b_{n}\left(\alpha_{n}\right)$ (see Fig. 1). Since $b_{n+1}(\alpha)<b_{n}(\alpha)$ and $b_{n}(\alpha) \rightarrow \infty$ as $\alpha \rightarrow 1 / 2$, the sequence $\left\{\alpha_{n}\right\}$ is increasing and bounded above by $1 / 2$. Therefore, we conclude that the $n$th CSS solution from section 3 is regular iff $\alpha<\alpha_{n}$. The first two values of $\alpha_{n}$ are $\alpha_{0} \approx 0.0688$ and $\alpha_{1} \approx 0.1588$.

\section{$5 \quad$ Final remarks}

The role of the CSS solutions considered in this paper in the dynamical evolution depends crucially on their stability with respect to small perturbations. Although we have not performed the stability analysis, it seems plausible that for sufficiently small $\alpha$ the stability properties are the same as for $\alpha=0$, that is the $n$th solution has exactly $n$ unstable modes. Speculations that follow are based on this assumption. We expect that for $\alpha<\alpha_{0}$ the situation is qualitatively the same as in the flat spacetime, that is the $n=0$ solution determines the universal asymptotics of a naked singularity formation and the $n=1$ solution sits at the threshold of this process. For $\alpha>\alpha_{0}$ the singularity of the $n=0$ solution is covered by a horizon so generic naked singularities presumably disappear - nota bene this could be interpreted as a gravitational desingularization phenomenon. In the range $\alpha \in\left(\alpha_{0}, \alpha_{1}\right)$ we expect the type II threshold behaviour with the $n=1$ CSS critical solution. What happens for $\alpha>\alpha_{1} \approx 0.1518$ ? Since the $n=1$ solution is not regular, it cannot be a bona fide critical solution (it can still appear as a local intermediate 
attractor, though). HLPTA showed in [5] that for large values of $\alpha$ the critical behaviour is DSS and the echoing period $\Delta$ increases rapidly as $\alpha$ tends to 0.18 from above (see Fig. 4 in [5]). Moreover, they noticed small deviations from exact DSS at this smallest $\alpha$ value for which they observed DSS critical collapse. These facts led them to conjecture that a critical solution ceases to be DSS for still smaller values of $\alpha$. This conjecture fits nicely with our conjecture that a critical solution ceases to be CSS for $\alpha>\alpha_{1}$. Taken together, these conjectures suggest that the transition from CSS to DSS critical behaviour is not sharp but rather has a crossover character. HLPTA are currently running the computer simulations of the critical behaviour for the coupling constants $\alpha<0.18$, so the above speculations should be verified (or falsified) soon. Hopefully, the numerical phenomenology will also help understand a yet unknown mechanism of the CSS/DSS changeover.

\section{Acknowledgments}

The research of PB was supported in part by the KBN grant 2 P03B 01016 . PB thanks Peter Aichelburg and his coworkers for discussions and sharing their numerical results with him.

\section{References}

[1] B. J. Carr and A. A. Coley, Self-similarity in general relativity, gr-qc/9806048.

[2] P. Bizoń, Equivariant self-similar wave maps from Minkowski spacetime into 3-sphere, mathph/9910026, to appear in Commun. Math. Phys.

[3] P. Bizoń, T. Chmaj, and Z. Tabor, Dispersion and collapse of wave maps, math-ph/9912009, to appear in Nonlinearity.

[4] S. L. Liebling, E. W. Hirschmann, and J. Isenberg, Critical phenomena in nonlinear sigma models, math-ph/9911020.

[5] S. Husa, C. Lechner, M. Pürrer, J. Thornburg, and P. C. Aichelburg, Type II critical collapse of a self-gravitating nonlinear $\sigma$-model, gr-qc/0002067.

[6] S. Husa, C. Lechner, M. Pürrer, J. Thornburg, and P. C. Aichelburg, private communication.

[7] C. Gundlach, Understanding critical collapse of a scalar field, Phys. Rev. D55, 695-713 (1997).

[8] D. M. Eardley, E. W. Hirschmann, and J. H. Horne, $S$ duality at the black hole threshold in gravitational collapse, Phys. Rev. D52, R5397-R5401 (1995). 


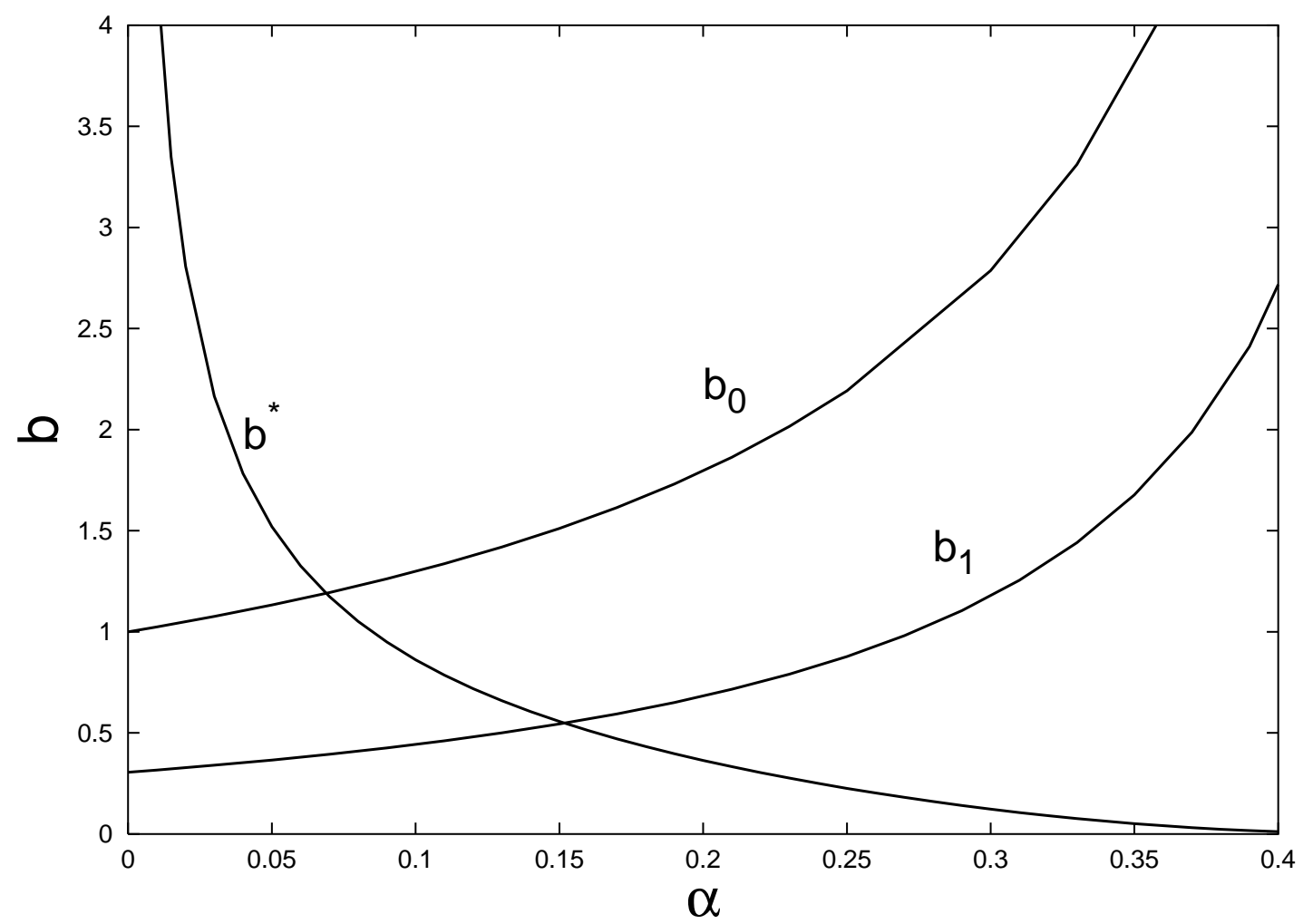

Figure 1: The shooting parameters $b_{n}(\alpha)$ for $n=0$ and $n=1$. The critical curve $b^{*}(\alpha)$ separates solutions that have an apparent horizon beyond the past SSH from those that do not. The intersections of the curve $b^{*}(\alpha)$ with the curves $b_{0}(\alpha)$ and $b_{1}(\alpha)$ determine the critical values of the coupling constant $\alpha_{0} \approx 0.0688$ and $\alpha_{1} \approx 0.1518$. 

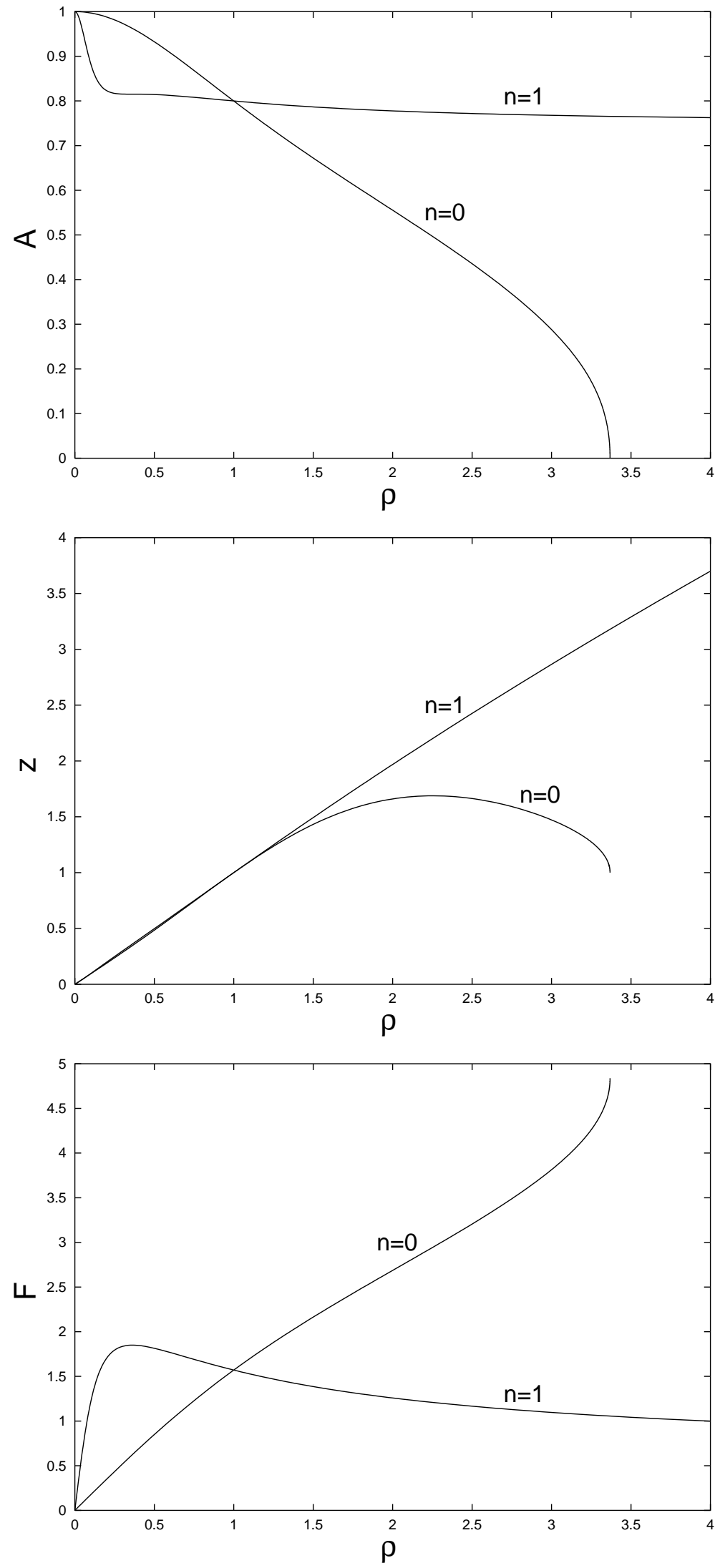

Figure 2: The $n=0$ and $n=1$ CSS solutions forl $\alpha=0.1$. Since $\alpha_{0}<0.1<\alpha_{1}$, the $n=1$ solution exists for all $\rho>1$, whereas the $n=0$ solution develops an apparent horizon at $\rho_{B} \approx 3.3683$. 

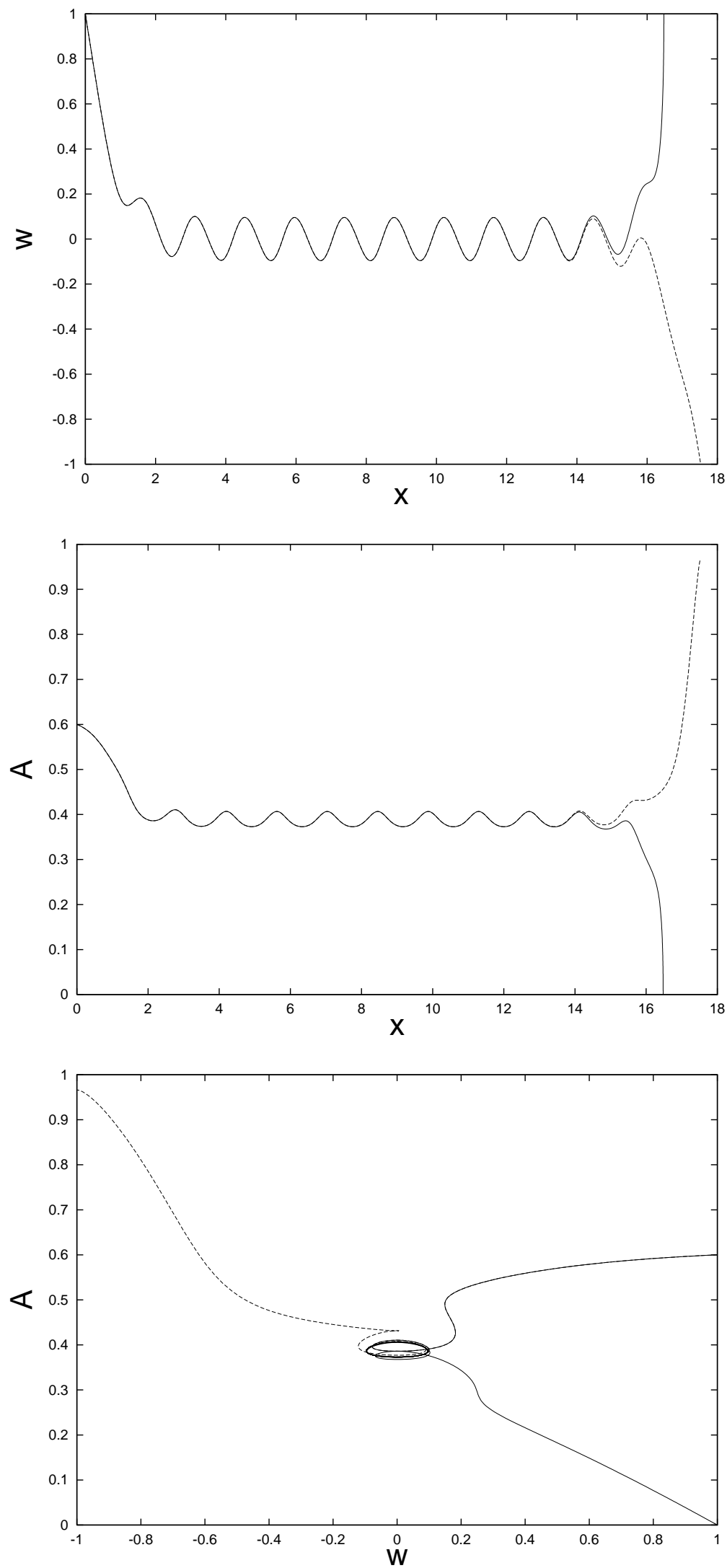

Figure 3: The metric functions $w$ and $A$ for two nearly critical solutions for $\alpha=0.2$ : type A solution with $b=0.36425022396604$ (dashed line) and type B solution with $b=0.36425022396605$ (solid line). For intermediate values of $x$ the solutions are almost periodic with the period $T \approx 1.418$. The intermediate attractor is seen on the $(w, A)$ plane as an unstable limit cycle. 


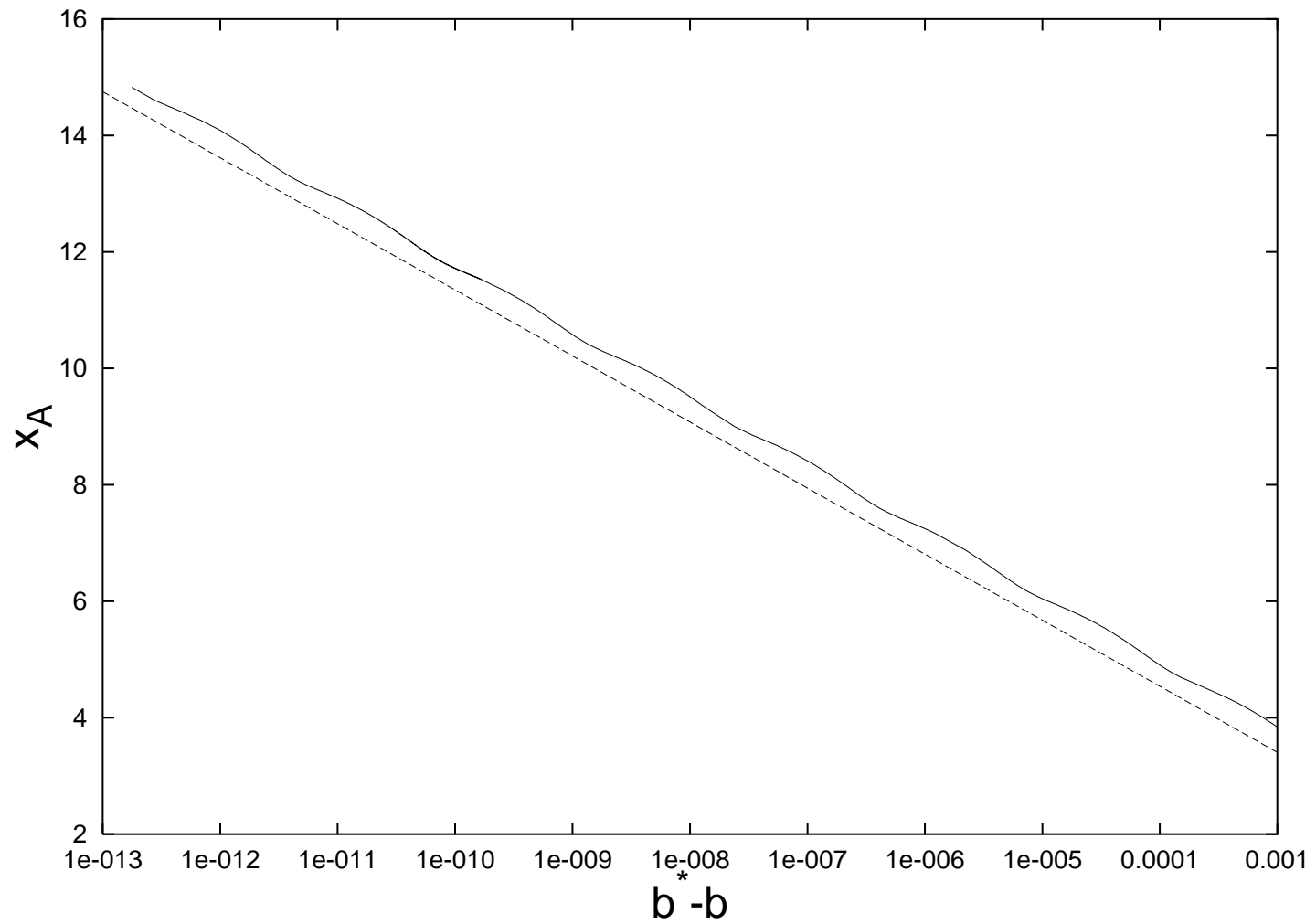

Figure 4: The locus of the future $\mathrm{SSH}, x_{A}$, against the logarithmic distance from the critical value, $\ln \left(b^{*}-b\right)$, for $\alpha=0.2$ is plotted as the solid line. The dashed line (shifted down for better comparison) shows the least-square fit of the leading order behaviour $x_{A}=-\frac{1}{\operatorname{Re}(\lambda)} \ln \left(b^{*}-b\right)$ with $\operatorname{Re}(\lambda) \approx 2.029$. The wiggles superimposed on this straight line are the imprint of periodicity of the intermediate attractor. The numerically computed period of the wiggles is approximately equal to 2.887 which agrees with the theoretically predicted value $\operatorname{Re}(\lambda) T$. 